\title{
Radio over fiber system based on a hybrid link for next generation of optical fiber communication
}

\author{
K. S. Alaoui ${ }^{1}$, J. Foshi ${ }^{2}$, Y. Zouine ${ }^{3}$ \\ 1,2LEIMP: Laboratory Electronics, Instrumentation and Measurement Physics, \\ Faculty of Science and Technology, Morocco \\ ${ }^{3}$ ISET Laboratory, National School of Applied Sciences ENSA of Kenitra, Ibn Tofail University Kenitra, Morocco
}

\begin{tabular}{l} 
Article Info \\
\hline Article history: \\
Received Nov 12, 2018 \\
Revised Mar 6, 2019 \\
Accepted Mar 12, 2019 \\
\hline
\end{tabular}

\section{Keywords:}

Access network OFDM

Radio over fiber SAC-OCDMA

\begin{abstract}
Radio over fiber technology will play an important role in solving problems facing wireless technology. Envisaging a global village, people could transmit and receive "anytime, anywhere, and anything". In addition, the explosive growth in internet applications such as the World Wide Web, demonstrates the tremendous increase in bandwidth and low power that the coming world of multimedia interactive applications will require from future networks. ROF technology uses multicarrier modulation like orthogonal frequency division multiplexing (OFDM), which provides an opportunity of having an increased in bandwidth together with an affordable cost and this idea has recently become a suitable topic for many research works. On the other hand, SAC-OCDMA (Spectral Amplitude Coding Optical Code Division Multiple Access) technique is able to enhance the data rate of system and increase the number of user. In this paper we introduce a ROF link using a hybrid OFDM/SAC-OCDMA technique.
\end{abstract}

Copyright (c) 2019 Institute of Advanced Engineering and Science. All rights reserved.

\section{Corresponding Author:}

K. S. Alaoui,

LEIMP: Laboratory Electronics, Instrumentation and Measurement Physics,

Faculty of Science and Technology,

Errachidia, Morocco.

Email: kaoutarsaidi@gmail.com

\section{INTRODUCTION}

The next generation of cellular mobile phone systems will make extensive use of microcells. This will permit a large increase in the numbers of users and will also allow a significant increase in the available channel bandwidth, so that broadband services can be offered, in addition to the voice band services offered with current systems.

The introduction of large numbers of microcells will result in the need to interconnect huge numbers of cells and microcells, and this can be carried out effectively using optical fiber, which offers a high transmission capacity at low cost [1]. Radio over fiber (RoF) systems have many advantages and applications as shown in Table 1 such as:

Table 1. Advantages and applications of RoF technology

\begin{tabular}{lll}
\multicolumn{1}{c}{ Advantages } & \multicolumn{1}{c}{ Indoor } & \multicolumn{1}{c}{ Outdoor } \\
\hline - Enhanced microcellular & - Airport terminals & - Underground \\
coverage & - Shopping centers & - Tunnels \\
- Higher capacity & - Large offices, & - Narrow streets \\
- Lower cost & & - Highways \\
- Lower power & & \\
- Easier installation & & \\
\hline
\end{tabular}


ROF technology uses multicarrier modulation like orthogonal frequency division multiplexing (OFDM), which offers an opportunity of increasing a bandwidth together with a reasonable cost and this idea has recently become a suitable topic for many research works [2-3]. Due to its less Inter Symbol Interference (ISI), computational complexity and more robustness as compared to other multiplexing schemes and Orthogonal Frequency Division Multiplexing (OFDM) has been extensively used in wireless communication. OFDM dominated in wireless broadcast system such as Wi-Fi and WiMAX because of its robustness to multipath fading and high sub-carrier density through the digital FFT and IFFT [4].

Optical code-division multiple-access (OCDMA) techniques are also suggested to be a more flexible solution in optical local area networks because multiple users are able to access the network asynchronously and simultaneously. In recent years, spectral amplitude coding (SAC) scheme of optical CDMA has been introduced to eliminate the MAI (multiple access interferences) effect and preserve the orthogonality between the users in the system. Several quasi-orthogonal code families are used in such spectral amplitude coded optical CDMA (SAC-OCDMA) systems. In this paper we introduce a Radio over fiber link using a hybrid OFDM/SAC-OCDMA technique to achieve a high- speed data rate and large bandwidth.

\section{RELATED WORKS}

A brief survey of literature in the area relevant to this paper is as follows. To Increasing Capacity and Suppress the crosstalk Aldhaibani et al. [5] proposed a Hybrid optical OFDM/WDM system with 10Gb/s downstream for different modulation. Sahbudin et al. [6] proposed the performance of SAC-OCDMA over the hybrid of FTTx-FSO network for the last mile users, The results reveal that the proposed SAC-OCDMA FTTX-FSO could support the maximum FSO range of 5.56, 3.6, 2.33, and $1.48 \mathrm{~km}$ for drizzle, light rain, medium rain and heavy rain, respectively, at the acceptable BER of $10^{-9}$.

Talli and Townsend [7] proposed Hybrid DWDM-TDM long-reach PON for next-generation optical access. Pehraj et al. [8] have shown that the requirement of high bandwidth, high flexibility, high mobility and high data rate at lower cost can be met with a real convergence of radio over fiber (RoF) and orthogonal frequency division multiplexing (OFDM) techniques. To increase the bandwidth Alateeq et al. [9] present a Design of Broadband RoF PON for the Last Mile, Zhang et al. [10] proposed a novel architecture for nextgeneration passive optical network (PON) based on electrical code division multiplexing orthogonal frequency division multiplexing (ECDM-OFDM) access. Aldhaibani et al. [11] presented a 2.5-Gb/s hybrid WDM/TDM downstream link, using radio-over-fiber (RoF) techniques in the GPON network, where 16QAM is used at $2.4 \mathrm{GHz}$ frequency. The result shows a good performance with a distance of $25 \mathrm{~km}$ for single mode fiber which serves up to 32 users, [12] they also showns that an Orthogonal frequency division multiplexing (OFDM) combined with spectral amplitude coding optical code division multiple access (SACOCDMA) is a new technology investigated. The data rate and distance in SAC-OCDMA System has been developed.

Sahbudin et al. [13] proposed a performance improvement of hybrid subcarrier multiplexing optical spectrum code division multiplexing system using spectral direct decoding detection technique. Noweir et al. [14] proposed a cost-effective and compact architecture of an RoF fronthaul link for future 5G mobile C-RANs.

\section{RADIO OVER FIBER TECHNOLOGY}

Radio-over-Fiber technology entails the use of optical fiber links to distribute radio frequency signals from a central location (headend) to Remote Antenna Units (RAUs). In narrowband communication systems and WLANs, RF signal processing functions such as frequency up-conversion, carrier modulation, and multiplexing, are performed at the BS or the RAP, and immediately fed into the antenna. RoF makes it possible to centralize the RF signal processing functions in one shared location (headend), and then to use optical fiber, which offers low signal loss $(0.3 \mathrm{~dB} / \mathrm{km}$ for $1550 \mathrm{~nm}$, and $0.5 \mathrm{~dB} / \mathrm{km}$ for $1310 \mathrm{~nm}$ wavelengths $)$ to distribute the RF signals to the RAUs, as shown in Figure 1 [1].

\section{OFDM}

OFDM is used extensively in broadband wired and wireless communication systems because it is an effective solution to inter Symbol interference (ISI) caused by a dispersive channel. Very recently, a number of papers have described the use of OFDM in a range of optical systems including optical wireless, multimode fiber and single mode fiber [15, 16, 17]. In practice, OFDM systems are applied using a combination of Fast Fourier Transform (FFT) and Inverse Fast Fourier Transform (IFFT) blocks as shown in Figure 2. OFDM dominated in wireless broadcast system such as Wi-Fi and WiMAX because of its robustness to multipath fading and high sub-carrier density through the digital FFT and IFFT [4, 18]. 


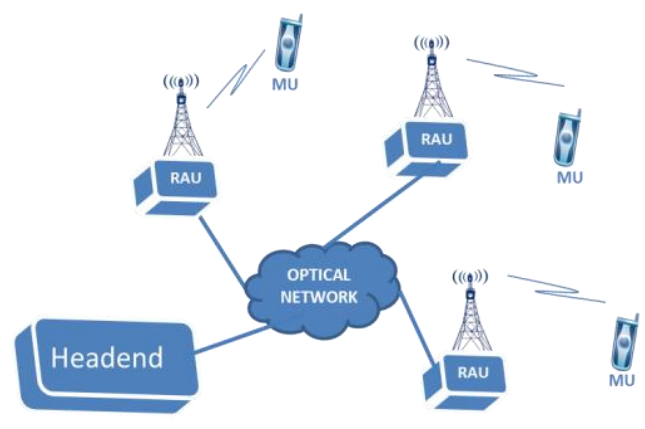

Figure 1. The radio over fiber system concept (RAU: remote antenna units, MU: mobile units)

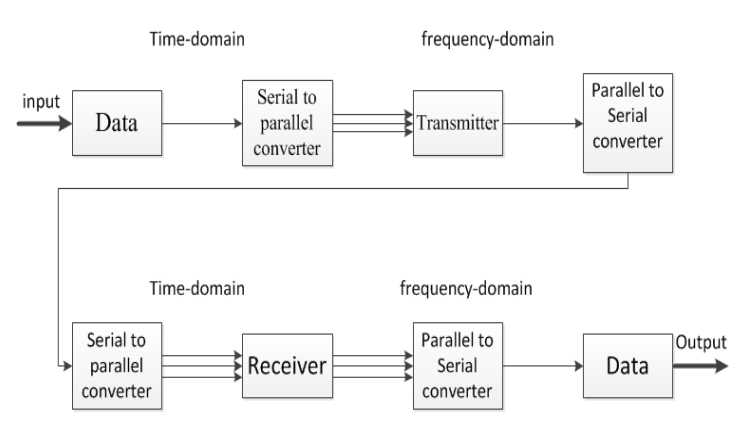

Figure 2. Block diagram of a simple OFDM system

\section{SAC-OCDMA}

In OCDMA systems, each user is given one code word for distinctness. The main factor of performance degradation in optical code-division multiple-access (CDMA) systems is the multiuser access interference (MAI), as the number of users increasing the performance of OCDMA system reduce because the (MAI), interference [19]. In spectral-amplitude- coding (SAC) systems, MAI is solely a function of the in-phase cross correlations among the address sequences (also known as signature sequences or spreading sequence or simply code sequences). If the in-phase cross correlation among the address sequences is fixed, then the balanced detection receiver is able to suppress MAI completely.

In SAC-OCDMA systems, each user is assigned with a sequence code that serves as its address. A user modulates its code with each data bit and asynchronously initiates transmission. Thus, this modifies its spectrum appearance, in a way recognizable only by the intended receiver Figure 3 . Let $\lambda=\sum_{i=1}^{N} \quad x_{i} y_{i}$ as the in-phase cross correlation of two different sequences $\mathrm{X}=\left(x_{1}, x_{2}, \ldots, x_{N}\right)$ and $\mathrm{Y}=\left(y_{1}, y_{2}, \ldots, y_{N}\right)$. A code with length $\mathrm{N}$, weight $\mathrm{w}$ and in-phase cross correlation $\lambda$ can be denoted by $(\mathrm{N}, \mathrm{w}, \lambda)[20]$. The code is considered possess an ideal in-phase cross correlation when $\lambda=1$. The balanced detection receiver is able to suppress MAI completely, provided that the family of codes used satisfies the following property: $\lambda_{X Y}=\lambda_{\underline{X Y}}$, $\lambda_{X Y}$ is the in-phase cross-correlation function between the codes of the desired user $\mathrm{X}$ and that of an undesired user $\mathrm{Y} . \underline{X}$ is a sequence complementary to $\mathrm{X}$.

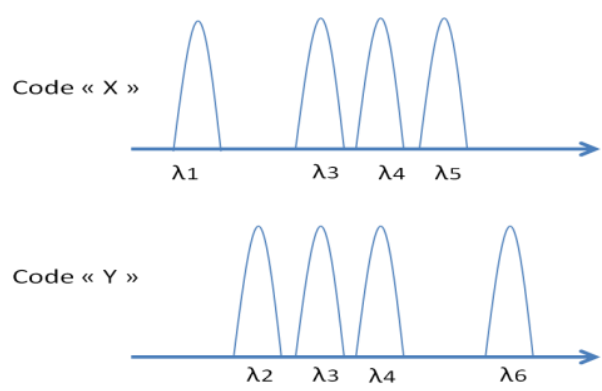

Figure 3. Codes example for SAC-OCDMA

\section{SYSTEM DESIGN}

The system design consists of three main parts which are the transmission part, the transmission link and the receiver part. Figure 4 shows the block diagram of the Radio over Fiber link using hybrid OFDM/SAC-OCDMA system. The proposed configuration combines SAC-OCDMA technology and OFDM. For the transmission part the OFDM signal based on 16-QAM is generated. The OFDM subcarriers with independent unipolar digital signal are optically modulated onto the code sequence using an optical modulator. The data streams are orthogonal to each other in both code chips and subcarriers, which cannot only eliminate the crosstalk between the sub-channels, but also ensure the security of the data. In RoF link using hybrid OFDM/SAC-OCDMA system, each user is allocated with one sub-channel including one specific code chip and one or more subcarriers. The subcarriers can be used for different services, such as voice/video signal, point to point $(\mathrm{P} 2 \mathrm{P})$ and WiMax signals. Then the modulated code sequences are combined together and transmitted through the optical fiber link. 


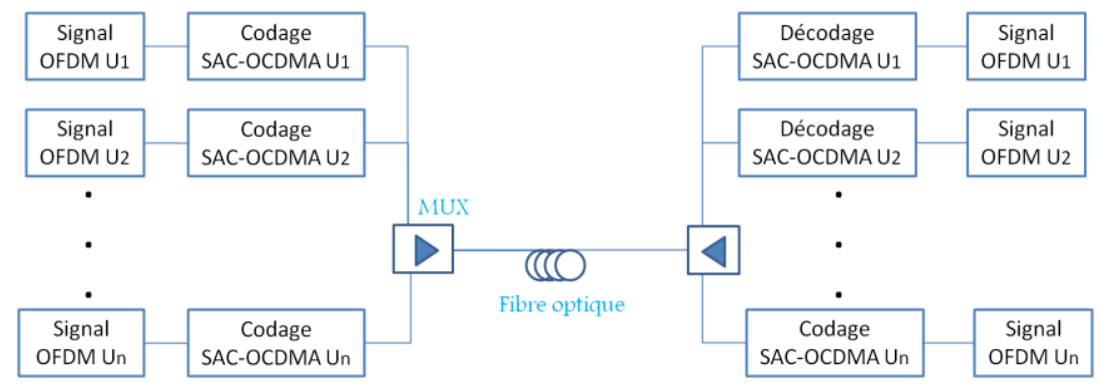

Figure 4. The block diagram of the RoF link using hybrid OFDM/SAC-OCDMA

At the receiver, an optical splitter is used to separate the different modulated code sequences to different optical network users. Then, the resulting signal is detected by the photodetector to convert optical signal to electrical signal, OFDM receiver module which decodes an electrical QAM-OFDM signal as generated by transmitter block.

The proposed configuration, shown in the Figures 5 and 6, contains the Optical transmitter situated at the Headend or Central Office (CO) and several Remote Antenna Units, comprises an Optical Network Units (ONUs) and antennas, located at some particular distant location. Here the Optical transmitter comprises the fundamental blocks of RF OFDM transmitter as shown in Figure 7, a RF-to-Optical (RTO) upconverter and encoder SAC-OCDMA as shown in Figure 8 to encode each user's data with an appropriate code. And after passing through the optical link each ONU includes an optical-to-RF (OTR) down-converter and a RF OFDM receiver.

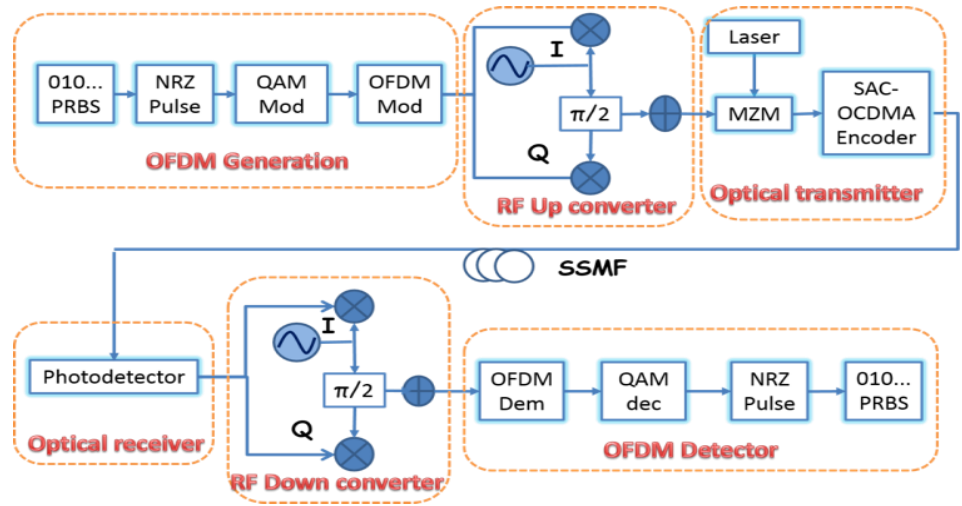

Figure 5. The proposed configuration combines SAC-OCDMA and OFDM

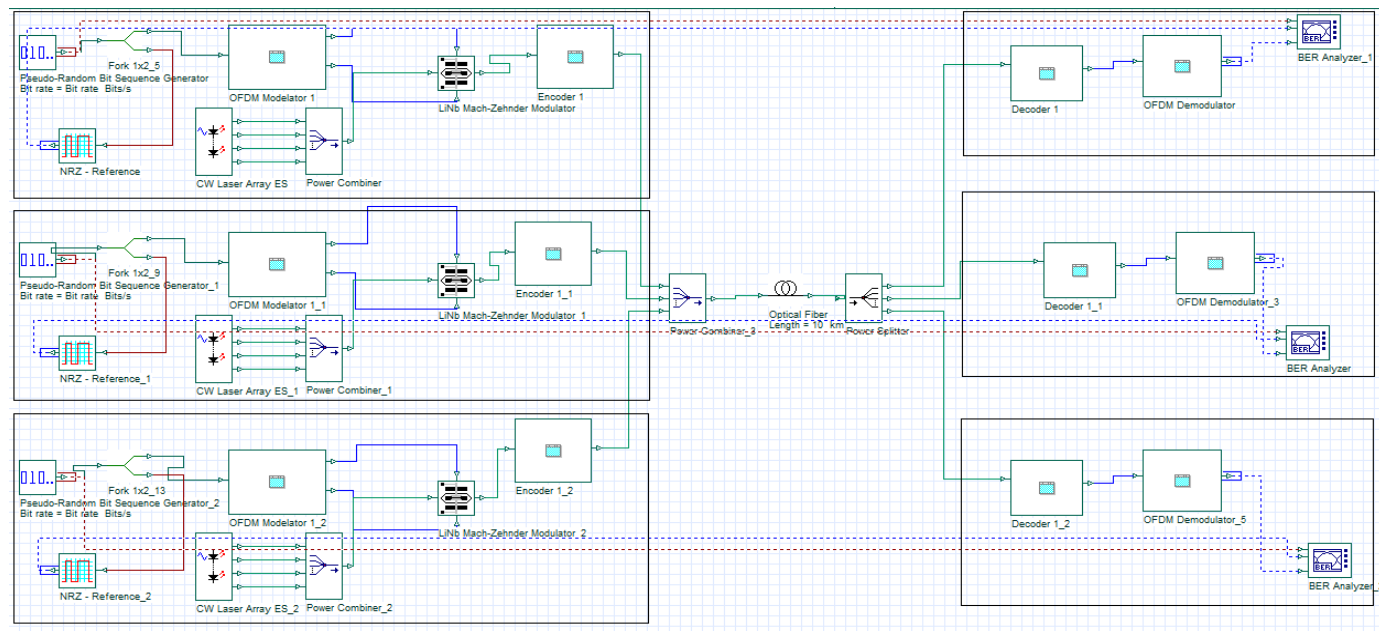

Figure 6. The propose system architecture 


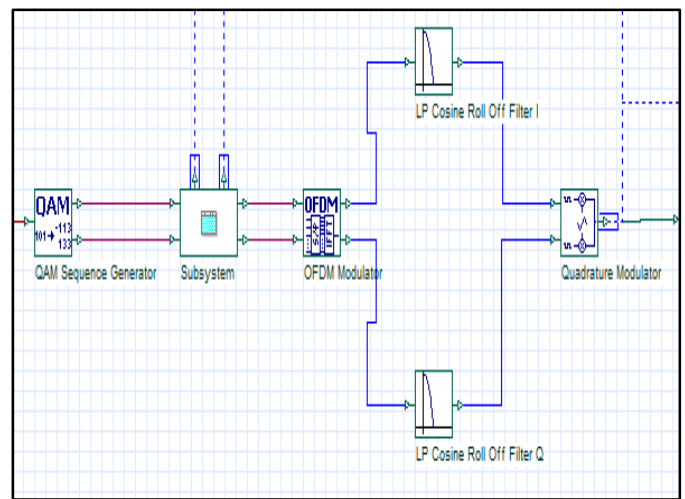

Figure 7. OFDM Modulator

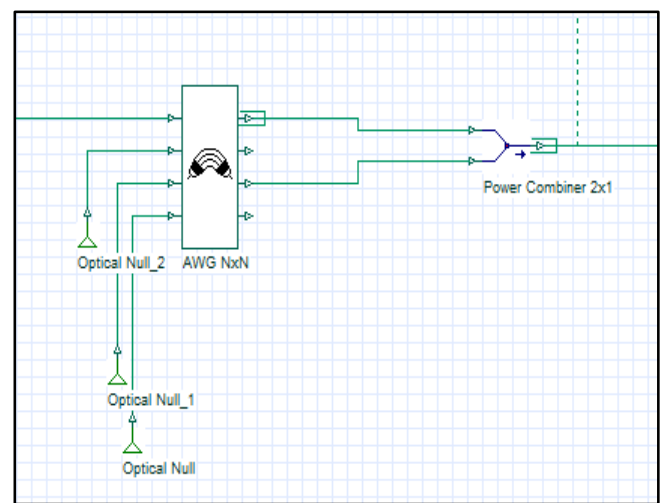

Figure 8. Encoder SAC-OCDMA

In the RF OFDM transmitter, the input digital data are first converted from serial to parallel block of bits consisting of information symbol where each symbol comprise multiple bits of M-ary coding. And, in our proposed model we used M-ary QAM for constellation. The time domain OFDM signal is obtained through Inverse Fast Fourier Transform (IFFT). The baseband OFDM signal can be up-converted to a RF pass band through a RF IQ mixer. The subsequent RTO up-converter transforms the baseband signal to the optical domain using an optical IQ modulator comprising a Mach- Zehnder-modulator (MZM) with a 90degree phase offset. Then finally the optical signal is transmitted through a standard single mode fiber (SSMF). At the receiving end the optical signal travelling through the SSMF is converted back to RF OFDM signal by the OTR down-converter. The received RF signal is detected by a PIN photodetector which converts the optical signal to an electrical one. Besides the PIN photodetector the ONU also contains a digital I-Q demux, followed by an FFT.

Table 2 show the Bipolar Walsh Hadamard code with a code length of 4 and showing which wavelength should be chosen.

Table 2. Walsh Hadamard codes

\begin{tabular}{cc}
\hline Walsh Hadamard codes for 3 Users & Chosen wavelength \\
\hline 1010 & $\lambda_{1} \lambda_{3}$ \\
1100 & $\lambda_{1} \lambda_{2}$ \\
1001 & $\lambda_{1} \lambda_{4}$ \\
\hline
\end{tabular}

Knowing that: $\lambda_{1}=193.1 \mathrm{Thz}, \lambda_{2}=193.2 \mathrm{Thz}, \lambda_{3}=193.3 \mathrm{Thz}, \lambda_{4}=193.4 \mathrm{Thz}$.

The Figure 9 show the different optical carriers emitted by the lasers and the user's optical signal after SAC-OCDMA encoding.

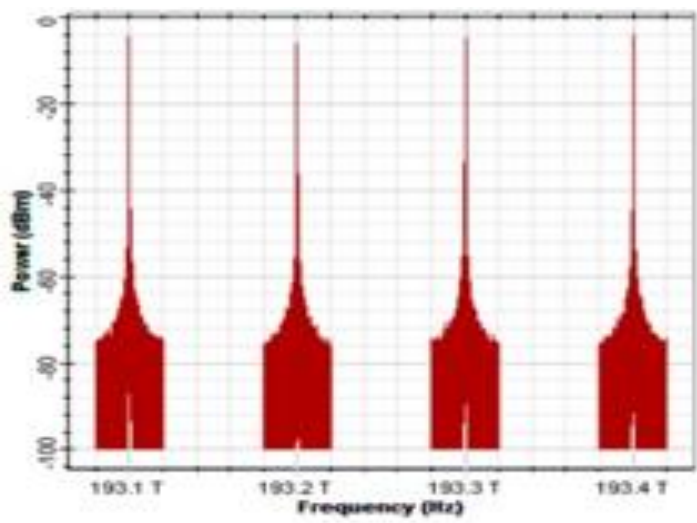

(a)

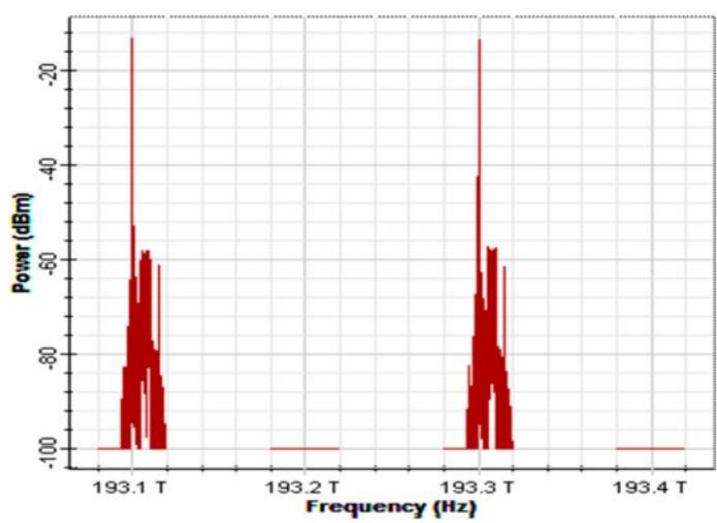

(b)

Figure 9. (a) The different optical carriers emitted by the lasers (b) The first user optical signal after SAC-OCDMA encoding 


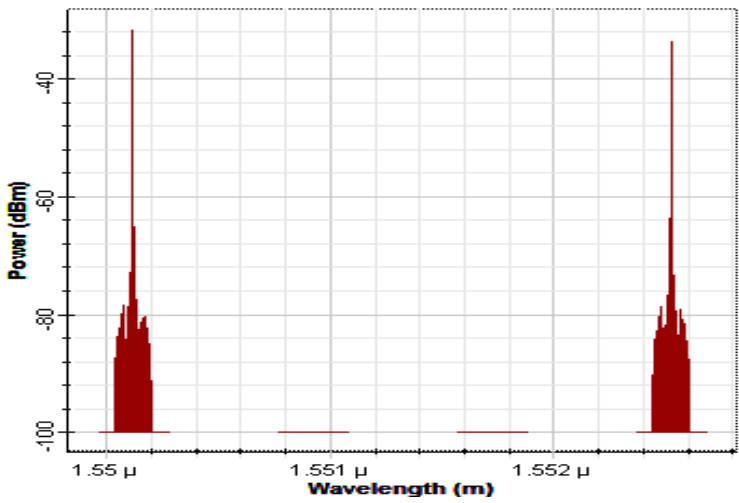

(c)

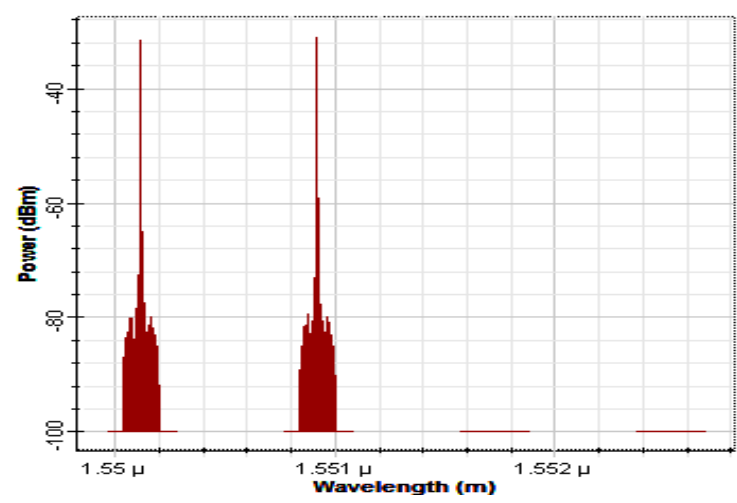

(d)

Figure 9. (c) The second user optical signal after SAC-OCDMA encoding

(d) The third user optical signal after SAC-OCDMA encoding

On reception, the photodetector detects the light and transforms it into an electrical signal before demodulating the signal. The low-pass filter has been set up to eliminate the high frequencies. Thereafter the amplifier limited in gain for the purpose of amplifying the signal, finally down to a baseband signal but it is noisy, the latter comes from the electronic and optoelectronic components. To reduce the impact of this noise, another low-pass filter is used. The received OFDM signal after decoding and the first user's diagram of the eye. It is visualized that the eye diagram is open which means that there is no interference between symbols, the bit error rate is $1.8^{*} 10^{-10}$.

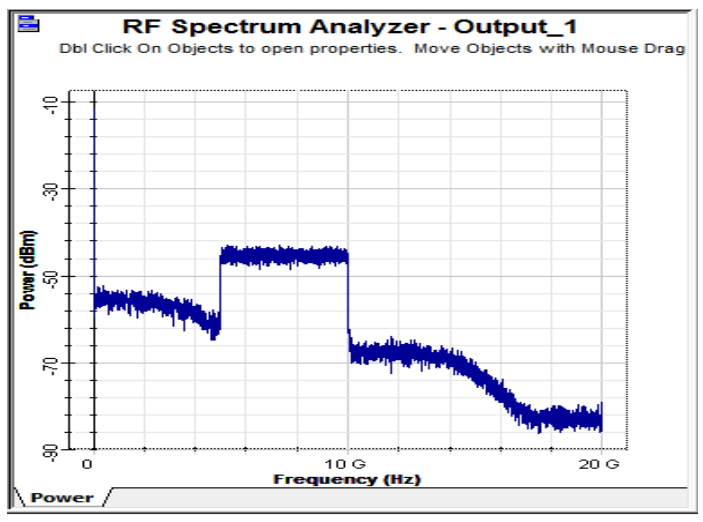

(a)

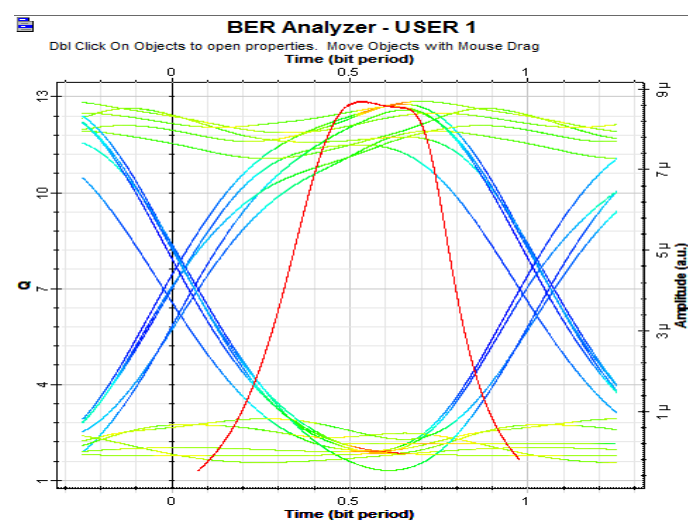

(b)

Figure 9. (a) The received OFDM signal after decoding (b) Diagram of the eye user 1

\section{CONCLUSION}

In this paper we proposed a hybrid radio over fiber link based on various modulation schemes and simulated through a fiber optic. Previous research works related to PON derived bit error rate (BER) from the measured vector magnitude and compared the performance among different modulation techniques but never showed the performance for both OFDM and SAC-OCDMA together. But in our paper we investigate the performances of ROF link using a hybrid OFDM/SAC-OCDMA technique.

\section{REFERENCES}

[1] D. Wake, "Radio over Fiber Systems for Mobile Applications," Radio over Fiber Technologies for Mobile Communications Networks, in H. Al-Raweshidy and S. Komaki, ed., Artech House, Inc, USA, 2002.

[2] Y. M. Lin, "Demonstration and design of high spectral efficiency 4Gb/s OFDM system inpassive optical networks," Proc. of OFC, Anaheim, USA, Paper OThD7, 2007. 
[3] T. Duong, et al., "Low cost multi band-OFDM for remote modulation of colourless ONU in hybrid WDM/TDMPON architecture," Proc. of ECOC, Berlin, Germany, 2007.

[4] S. Hara, "Multicarrier Techniques for 4G Mobile Communications," Norwood, MA: Artech House, Incorporated, 2003.

[5] A. O. Aldhaibani, et al., "Increasing Capacity and Suppress The Crosstalk by Using Hybrid Optical OFDM/ WDM System,” Key Engineering Materials, vol. 594-595, pp. 1041-1044, 2014.

[6] R. K. Z. Sahbudin, et al., "SAC-OCDMA over Hybrid FTTx Free Space Optical Communication Networks," Journal of telecommunicationsand information technology, pp. 52-58, 2015.

[7] G. Talli and P. D. Townsend, "Hybrid DWDM-TDM long-reach PON for next-generation optical access," $J$. Lightwave.Technol, vol. 24, pp. 2827-2834, 2006.

[8] Pehraj and Divya, "Performance Analysis of RoF PON System based on Orthogonal Frequency Division Multiplexing Technique," International Journal of Computer Applications, vol/issue: 95(26), 2014.

[9] A. Alateeq, et al., "Design of Broadband RoF PON for the Last Mile," Communications and Network, vol. 4, pp. 269-277, 2012.

[10] L. Zhang, et al., "A novel ECDM-OFDM-PON architecture for Next-Generation optical access network," Optical Society of America, vol/issue: 18(17), 2010.

[11] A. O. Aldhaibani, et al., "Hybrid wavelength division multiplexing/time domain multiplexed (WDM/TDM) using radio over fiber technique with 16QAM at 2.5 Gbps," International Journal of Physical Sciences, vol/issue: 8(18), pp. 897-900, 2013.

[12] A. O. Aldhaibani, et al., "Increasing performance of sac-ocdma by combine ofdm technique," Journal of Theoretical and Applied Information Technology, vol/issue: 66(2), 2014.

[13] R. K. Z. Sahbudin, et al., "Performance improvement of hybrid subcarrier multiplexing optical spectrum code division multiplexing system using spectral direct decoding detection technique," J. Optical Fiber Technol, vol. 15, pp. 266-273, 2009.

[14] M. Noweir and M. Helaoui, "Digitally Linearized Radio-Over Fiber Transmitter Architecture for Cloud Radio Access Network's Downlink," IEEE Transactions on Microwave Theory and Techniques.

[15] N. Nowshin, et al., "Demonstration and performance analysis of rof based ofdm-pon system for next generation fiber optic communication," International Journal of Computer Networks \& Communications (IJCNC), vol/issue: 4(1), 2012.

[16] M. K. Sasikala and D. Helena, "Multiuser Based on Subcarrier Multiplexing with Spacing of 5Ghz in Radio over Fiber System using Direct Detection," International Conference on Electrical, Information and Communication Technology (IJARI), 2015.

[17] P. Mittal, et al., "Coherent Detection Optical OFDM System," International Journal of Scientific Research Engineering \& Technology (IJSRET), vol/issue: 4(4), 2015.

[18] M. K. Samimi and T. S. Rappaport, "3-D millimeter-wave statistical channel model for 5G wireless system design," IEEE Trans. Microw. Theory Techn., vol/issue: 64(7), pp. 2207-2225, 2016.

[19] Anuar M. S., et al., "New design of spectral amplitude coding in OCDMA with zero cross-correlation," Optics Communications, vol/issue: 282(14), pp. 2659-64, 2009.

[20] Y. Zouine and Z. Madini, "Analyse des performances de plusieurs codes pour un système W-OCDMA d'un reseau EPON." 